\title{
Clinical and Pathological Studies on Feline Infectious Peritonitis in Egypt
}

\author{
Ahmad Zaki Anwer ${ }^{1}$, Mohamed R. Mousa ${ }^{2}$, Mouneir Abdel Halium ${ }^{1}$, Yara S. Abouelela ${ }^{3}$ and Hasan \\ M. Elsaid ${ }^{1 *}$
}
${ }^{1}$ Department of Medicine and Infectious Diseases, Faculty of Veterinary Medicine, Cairo University, Giza, Egypt ${ }^{2}$ Department of Pathology, Faculty of Veterinary Medicine, Cairo University, Giza, Egypt
${ }^{3}$ Anatomy and Embryology Department, Faculty of Veterinary Medicine, Cairo University, Giza, Egypt
*Corresponding author: hasanelsaid874@gmail.com

Article History: 21-404 Received: 18 -Sep-21 Revised: 28-Sep-21 Accepted: 15-Oct-21
ABSTRA C T
Feline infectious peritonitis (FIP) is a highly fatal multi-systemic disease of wild and domesticated Felidae which is
developed as a mutant type for the harmless feline enteric coronavirus (FeCoV). In Egypt, there is neither documented
data about feline infectious peritonitis nor feline enteric coronaviruses till now. The study was performed to highlight
on the clinical, pathological and some epidemiological findings for FIP diseased cats in Egypt. For this purpose, 169
cats ( 85 males and 84 females) of different breeds aged from 4 months to 8 years old were examined. Different
technical methods were used to confirm the diagnosis of the clinically suspected cases including Rivalta's test,
immunochromatographic tests, ultrasonography, radiography, hematological testing, histopathology, and RT-PCR.
Rapid immunoassay SNAP Combo tests were used to exclude viral infection causing resembling symptoms like feline
immune deficiency and/or feline leukemia viruses. Nearly $90 \%$ of the examined cats (152 out of 169) have been
identified as carriers for feline enteric coronavirus FeCoV. Typically, FIP clinical signs were observed only in the
multi-cat household especially in animal shelters. 9 cases were identified with FIP disease from total of 169; FIP was
nearly equal between both sexes (five males and four females) along with the higher incidence was observed in age
above two years old. Clinical signs associated with FIP were recorded only in cats of foreign breeds (6 Persian, 1
Himalayan and 2 mixed breed) and no FIP clinical cases were reported in the Egyptian Mau cats. Frequent incidence
of complicating diseases like FIP is considered a remarkable challenge for veterinarians especially in its diagnosis.
Therefore, more attention and studies are required to ease and confirm the diagnosis of such diseases with less specific
symptoms.

Key words: Feline infectious peritonitis, feline enteric coronavirus, cats, Egypt.

C202I IJVS - All Rights Reserved

\section{INTRODUCTION}

Feline infectious peritonitis (FIP) is a deadly immune mediated viral disease that affects wild and domestic cats. It arises because of the mutation of the avirulent feline enteric coronavirus $\mathrm{FeCoV}$ within a small percentage of infected cats (Michimae et al. 2010; Kipar and Meli 2014; Tekes and Thiel 2016; Howell et al. 2020). Both FeCoV and FIP are belonged to the same virus species feline coronavirus $\mathrm{FeCoV}$ but they are totally different distinct pathotypes (Howell et al. 2020). Infection with FeCoV is very common and about $40-50 \%$ of the domestic cat population has been infected with this type, this figure could increase to reach $90-100 \%$ in multi-cat household (Meli et al. 2004; Vogel et al. 2010). Asymptomatic FeCoV infection was previously believed to be confined to the intestinal tract, but it is well known that healthy infected cats with $\mathrm{FeCoV}$ can have further systemic spread (Meli et al. 2004; Barker et al. 2010; Porter et al. 2014; Felten and Hartmann 2019). Transmission of $\mathrm{FeCoV}$ is highly contagious in multi-cat environment and occurs via fecal oral route; in contrast, FIP is not infectious via the fecal-oral route and developed as a consequence for different gene mutation.

Different risk factors play an important role for the development of FIP disease such as stress level and overcrowding. All of these risks could accelerate the viral replication and generate new viral mutants (Kass and Dent 1995; Felten and Hartmann 2019). Furthermore, the host immune status plays an additional role as it is revealed that FIP cats usually suffered from $\mathrm{T}$ lymphocytes depletion (Haagmans 1996; Jolanda et al. 2005), but still

Cite This Article as: Anwer AZ, Mousa MR, Halium MA, Abouelela YS and Elsaid HM, 2022. Clinical and pathological studies on feline infectious peritonitis in Egypt. International Journal of Veterinary Science 11(2): 159167. https://doi.org/10.47278/journal.ijvs/2021.099 
the genetic mutation is the main risk factor as along each cycle of viral replication, numerous mutations have been evolved (Kiss et al. 2000; Pedersen 2009; Desmarets et al. 2016; Hora et al. 2016). These mutations have the responsibility for triggering the tropism switch from enterocytes to macrophages (Rottier et al. 2005).

Genomic variation has been detected between FCoV from FIP affected cats and the FCoV from healthy ones especially in the Open Reading Frame (ORF) 3abc (Chang et al. 2010; Pedersen et al. 2012; Borschensky and Reinacher 2014; Hora et al. 2016). Furthermore, mutation at the sites of $3 \mathrm{a}$ and $3 \mathrm{~b}$ genes could be employed in FIP pathogenesis as well (Balint et al. 2012; Dedeurwaerder et al. 2013). Mutations in Spike (S) gene of the FCoV particularly in the $\mathrm{S} 2$ region results in change of viral cell tropism (Chang et al. 2012; Heald-Sargent et al. 2012; Bank-Wolf et al. 2014; Jaimes and Whittaker 2018). All these mutation types allow the FIP virus to have the capability for replicating efficiently in macrophages (Dewerchin et al. 2005; Lewis et al. 2015).

Clinical signs due to infection with $\mathrm{FeCoV}$ may be transient and could be limited to a short episode of upper respiratory tract symptoms and/or diarrhea with vomiting especially in Kittens. Rarely, the virus itself can be responsible for severe acute or chronic symptoms, however, most of FeCoV infected cats are asymptomatic (Pedersen. 1995).

In natural infection, the exact duration between the mutation and development of FIP clinical signs is mostly unknown as it certainly depends on the immune status of the infected cat. Most likely, the FIP clinical signs start to appear few weeks to 2 years after the viral mutation (Hartmann 2005). The FIP clinical and pathologic findings are usually occurring as a result for the extensive vasculitis which subsequently lead to multiple organs failure (Hartmann 2005; Kipar et al. 2005; Michimae et al. 2010).

The pathology of FIP disease has been classified into two distinct clinical forms, wet form (effusive type) which is characterized by polyserositis, abdominal and/or thoracic effusion with vasculitis. The second form is the dry form (non-effusive type) which is characterized by severe pyogranulomatous reaction in the internal organs (Kipar et al. 2005; Addie et al. 2009). Rather than the clear distinction between the wet and the dry forms of the disease, there is proportion of cases presented as a mixed between these two forms (Drechsler et al. 2011; Kipar and Meli 2014). The clinical course of the dry form is usually taking longer duration than wet form (Kipar and Meli 2014). The less specific symptoms are prominent in many FIP cases, and this may be due to multiple organs involvement like kidneys, liver, eyes, and even the CNS may be affected. Therefore, list of differential diagnoses should include the FIP disease for any affected cats with non-specific symptoms like lethargy, anorexia with resistant fever and chronic weight loss (Hartmann 2005).

Infection with $\mathrm{FeCoV}$ in cats could be diagnosed through demonstrating the virus itself by electronmicroscopy or by genomic detection using reverse RTPCR assay. Such investigations haven't been used frequently except for detection of carriers as many healthy individuals could have $\mathrm{FeCoV}$ in their feces (Sparkes 2004; Sharif et al. 2010).
Identification of FIP disease is characterized by high level of controversy as it has been described as one of the most misdiagnosed diseases and many of challenges have been faced especially when confronting very sick and small aged patients (Norris et al. 2005). These difficulties may be resulted from the lack of specific clinical symptoms, the lack of characteristic biochemical abnormalities, and lack of highly sensitive and specific tests. Therefore, FIP diagnosis is depending upon assimilation of the history, hematology, and other supportive diagnostic tests, including serology and findings from imaging, tissue biopsies, histopathology, and PCR (Simons et al. 2005; Brown et al. 2009; Sharif et al. 2010). Therefore, the present study was conducted to draw attention for some epidemiological aspects about FIP disease in Egypt, its various clinical manifestations, and related histopathological findings with using of different methods for diagnosis.

\section{MATERIALS AND METHODS}

\section{Animals}

A total of 169 cats of different ages, breeds and sex were subjected for the study including 40 Egyptian Mau cats, 80 Persian, 19 Himalayan and 30 mixed breeds $(n=169)$. According to the age, 129 animals were above two years old and the other 60 cats were below this age. According to the sex, 85 males and 84 females were examined. All of the investigated animals were belonged to different Egyptian Shelters and several multi-cat households. Most of them were admitted to the educational hospital at faculty of veterinary medicine, Cairo University suffering from non-specific complains.

\section{Physical Examination}

Complete case history was recorded for all clinical cases including age, sex, breed, and condition of their growing environment (single or multi-cat environment) Physical examination was implemented with observation of the general health condition, inspection of different mucous membranes, skin, and the superficial lymphnodes. Clinical parameters were recorded involving rectal temperature and pulse rate. Abdominal palpation with chest and heart auscultation was performed for each inspected case.

\section{Samples and diagnostic methods}

1. Fecal swabs were collected from all inspected (169) cats for qualitative testing against $\mathrm{FeCoV}$ antigen. Immunochromatographic Antigen Rapid (FCoV) $\mathrm{Ag} / \mathrm{Ab}$ Test Kit (CAT. NO. RC1207DD, Bionote, Korea) was used according to the manufacturer instructions.

2. Whole Blood and serum samples from all examined cats were collected for RT-PCR assay and hematological testing including complete blood picture, total protein, albumin/globulin ratio plus liver and kidney function. Serum samples were used for testing against FCoV antibodies by immunochromatographic lateral flow rapid test.

FOR RT-PCR, the test was performed to amplify sub genomic mRNA of the highly conserved $\mathrm{M}$ gene (Simons et al. 2005). Total RNA was isolated according to the 
manufacturer instruction using QIAamp® RNA Blood Mini (Catalog no. 52304, Qiagen). Primers were selected from the highly conserved $M$ gene. The sequences for primers used in the RT-PCR assay were stated as follow, primer 212 (5'TAATGCCATACACGAACCAGCT'3), and primer 1179 (5'GTGCTAGATTTGTCTTCGGAC ACC'3). For reverse transcription, Verso one step RTPCR PCR kit (thermo scientific, Germany) was used according to the manufacturer instructions. The PCR cycling protocol consisted of $10 \mathrm{~min}$ incubation at $95^{\circ} \mathrm{C}$ followed by 30 cycles of $1 \mathrm{~min}$ denaturation at $95^{\circ} \mathrm{C}$, $1 \mathrm{~min}$ primer annealing at $62^{\circ} \mathrm{C}$ then $1 \mathrm{~min}$ for primer extension at $72^{\circ} \mathrm{C}$. The 30 cycles were succeeded by $10 \mathrm{~min}$ at $72^{\circ} \mathrm{C}$ and the reaction mixture was finally cooled to $4^{\circ} \mathrm{C}$. For analysis, $20 \mathrm{ml}$ of each PCR sample was analyzed by electrophoresis using a $1.5 \%$ TAE agarose gel (GibcoBRL Life Technologies) for $45 \mathrm{~min}$ at 100V. A 100bp molecular weight ladder (Invitrogen) was used to mark the size of the amplified PCR products. Amplified products were finally visualized by using of ethidium bromide staining and UV radiation. Fragments of $295 \mathrm{bp}$ were revealed for positive samples.

For detection of FCoV antibodies, an immunochromatographic lateral flow rapid test (product code. GDX34-1, Global DX, UK) was used for all serum samples and performed according to the manufacturer instruction.

To exclude concurrent infection with Feline Immune Deficiency Virus (FIV) and/or Feline Leukemia Virus (FLV), a SNAP Combo FeLV Ag/FIV Antibody rapid immunoassay (Product Code 502A.02, IDEXX laboratories) was performed for each serum sample according to the manufacturer instructions. It was used for simultaneous detection of feline leukemia virus (FeLV) antigen and feline immunodeficiency virus (FIV) antibody in feline serum (Levy et al. 2017).

1. Effusive fluid samples were collected to perform Rivalta's test. A transparent test tube was filled with about 7-8 $\mathrm{ml}$ distilled water, to which 1 drop of concentrated solution of acetic acid was added and mixed properly. One drop of the effusion fluid was carefully added on the surface. The Rivalta's test is defined as negative if the drop disappeared while the test defined as positive if the drop retained its shape, stayed attached to the surface or slowly floated down to the bottom of the tube, drop or jelly fish-like (Hartmann et al. 2003; Felten and Hartmann 2019).

2. Tissue samples were collected from the dead animals (nine cats) including heart, brain, lungs, spleen, lymph nodes and kidneys. All samples were placed in neutral buffered formalin (10\%) to be examined with H\&E staining. For histopathological investigation, tissue samples were sectioned, warmed, and placed in xylene jar which was replaced after 10-15 min with fresh one for another 10-15 min. Slides were kept in descending series of alcohol then washed and stained with Meyer's hematoxylin for 10-15 min. After washing, slides were immersed in fast acid alcohol for few seconds. The slides were washed and placed in $2 \%$ aqueous eosin for $2-5 \mathrm{~min}$ then put in ascending series for alcohol at least for 5 minute and finally in absolute ethanol: xylene mixture (1:1) for 5 min. The sections were cleared in xylene two times for 10-15 min. Cover slides were mounted on slides and dried till examined (Chauhan and Agarwal 2008).

Further diagnostic tools including radiography and ultrasound scanning were used for all clinically ill cats to investigate all internal organs using Sonoscape ultrasound M12v and X-ray (Allenger 100mA).

\section{RESULTS}

\section{Physical Examination}

The detected apparent clinical signs for the suspected FIP cases were related mainly to the wet form. The abdominal swelling was commonly noted by the owners and sometimes was confused with pregnancy. Flappy distended abdomen with fluctuation of fluid waves was observed in all FIP suspected cases during palpation (Fig.1a). Abdominal masses were palpated in all suspected cases, and this may be due to enlarged mesenteric lymph nodes, visceral adhesion and/or large sized pyogranulomas. Three cats were presented with dyspnea, mouth breathing and cyanotic mucous membranes, may be resulted from thoracic and pericardial effusion, muffled sound was confirming during the chest examination by stethoscope. Temperature was recorded high above $40^{\circ} \mathrm{C}$ in eight cases, the high temperature was refractory for antibiotics and was accompanied usually by anorexia and lethargy. Two cats were presented with keratitis and keratitic precipitate (Fig.1b). Symptoms of yellowish discoloration of mucous membranes and ear pinna were well recognized in three clinical cases (Fig.1c). Nodular firm cutaneous lesions (Fig.1d) and skin fragility syndrome were detected in one case. Aspiration of effusive samples revealed yellowish viscous turbid fluid, and this may be due to high protein content.

Screening with the immunochromatographic test for antigen detection in feline feces revealed that $90 \%(152$ out of 169) of all inspected cats are carrying feline enteric coronavirus (Fig. 2a). Most of them had no apparent clinical signs and about $20 \%$ of them only had selflimiting diarrhea ( 31 cases out of the 152 positive cases).

Screening for qualitative detection of antibodies in serum samples revealed that about $51 \%$ of inspected cases were seroconverted (86 out of 169) regardless the FIP development.

Screening with Combo FeLV Ag/FIV Antibody rapid immunoassay revealed that all inspected cats were negative for FIV antigen and FLV antibody in their serum samples (Fig. 2b).

Testing with mRNA PCR for whole blood samples collected from all animals revealed that eight out of nine cases (histopathological confirmation) were positive $(8 / 9=88.8 \%)$ and high specificity was represented as 150 cases were negative out of resting $160(93 \%)$.

Rivalta's test revealed that eight clinical cases were positive out of eleven cases presented with abdominal effusion. Highly sensitive result $(88.8 \%)$ was revealed as 
eight cases out of positive samples (9) were identified with RT-PCR and confirmed by histopathology while one sample was negative for Rivalta's but confirmed as FIP by histopathology. Strongly positive result appeared as floating drop or funnel with contact to the surface (Fig. 2c).

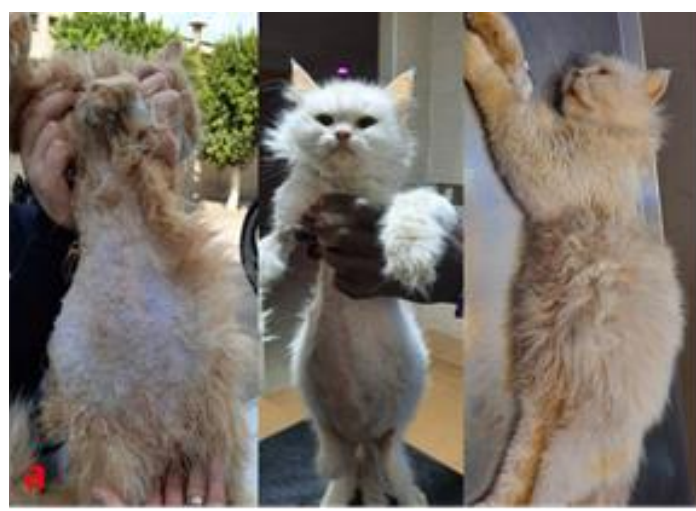

Hematological profile for FIP cases were commonly marked by lymphopenia, neutophilia, non-regenerative anemia, elevated ALT, creatinine and BUN. Variable results were observed in the total serum protein concentration, but the albumin/globulin ratio was detected below 0.8 for all cases.

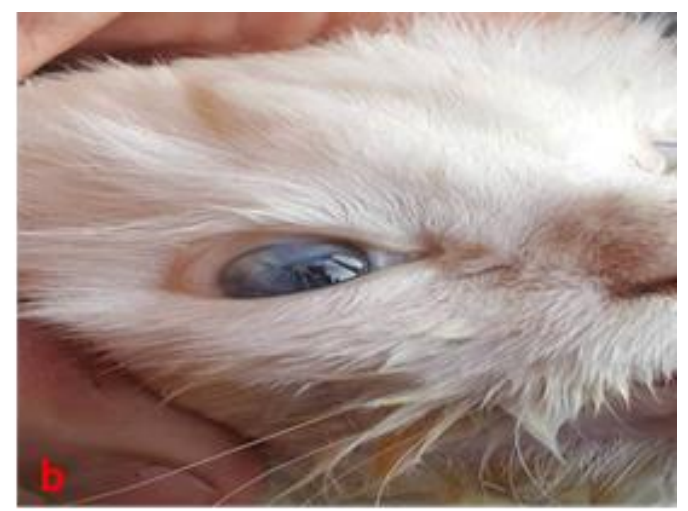

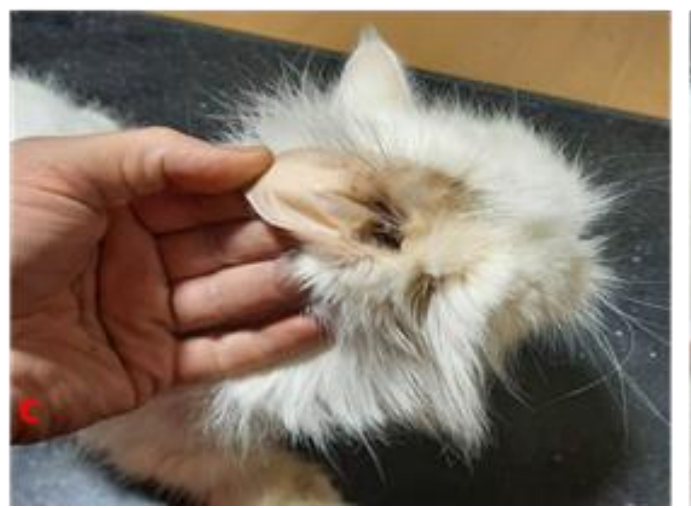

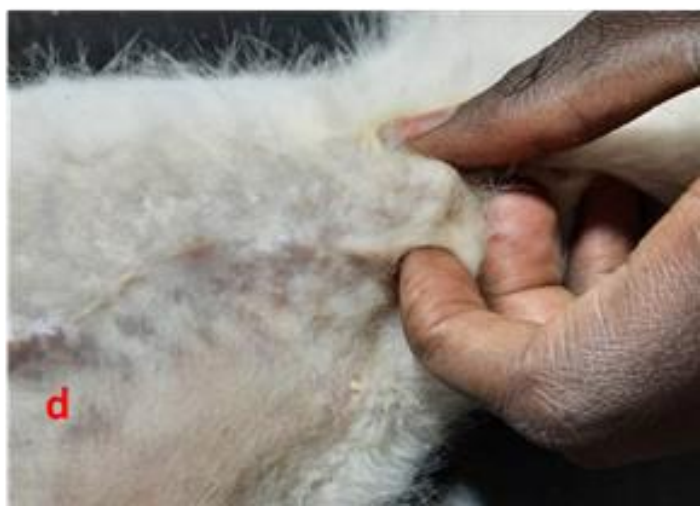

Fig. 1a: Abdominal distension in different cats affected with wet form of (FIP). b: Keratitic precipitate in right eye for a cat with (FIP). c: Yellowish discoloration of skin and ear pinna in FIP affected cat. d: Nodular skin lesions in cat with (FIP).
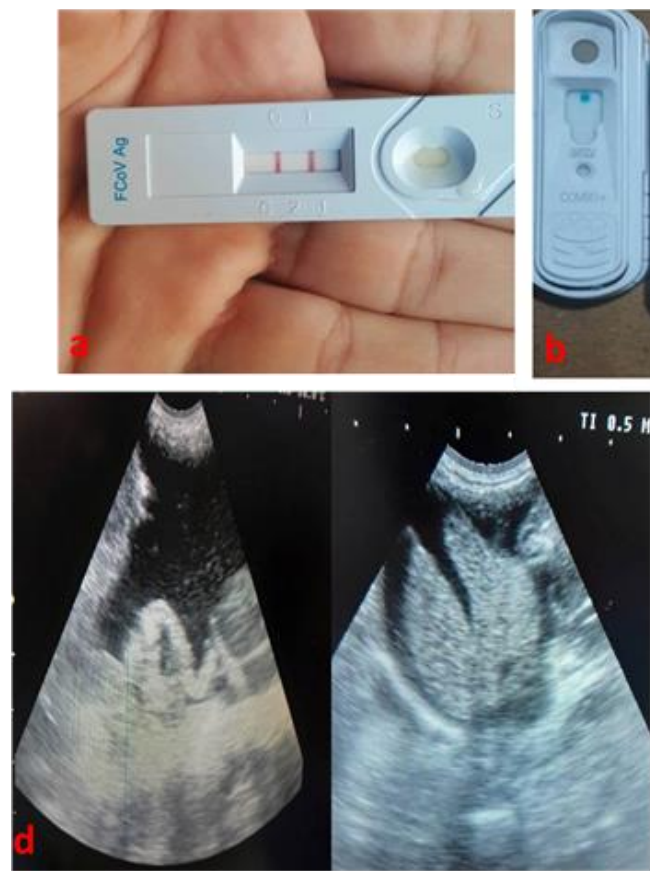
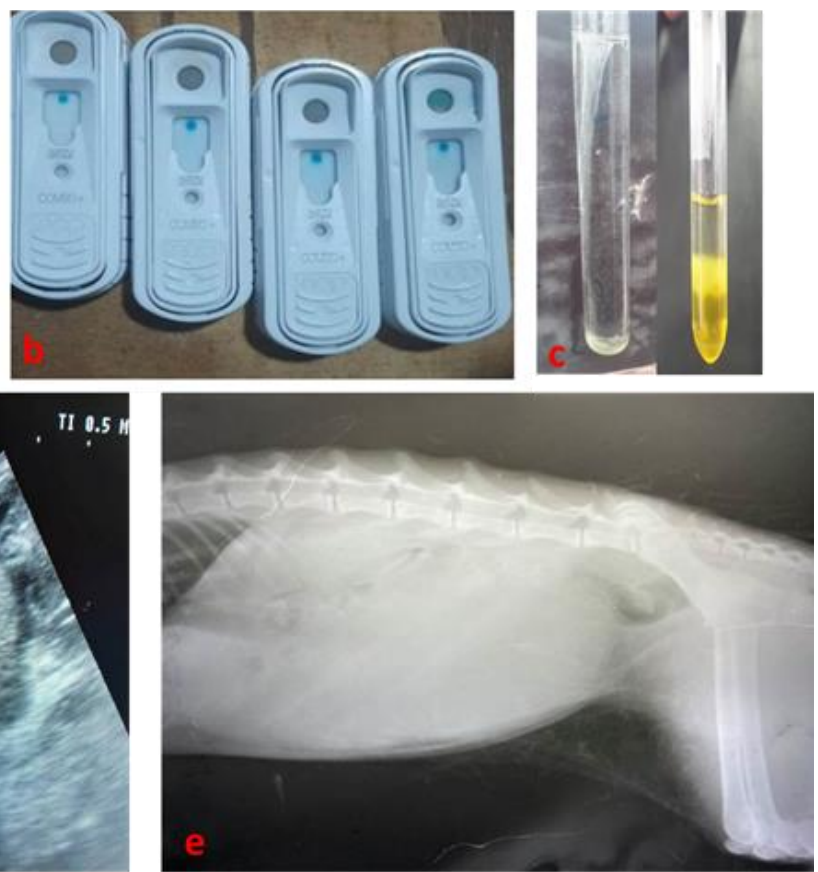

Fig. 2a: Immunochromatographic test showing positive result for feline corona antigen detection in feces. b: Combo SNAP rapid immunoassay showing negative results for FeLV Antigen ad FIV Antibody in serum samples for all tested cats. c: Rivalta's test showing positive result for effusive samples of cats with FIP. d: Ultrasonographic findings for FIP affected cat showing peritoneal effusion with marked changes in echogenicity for hepatic parenchyma. e: Radiography for FIP cat showing loss of serosal details due to peritoneal effusion. 
Diagnostic Imaging by ultrasound showed free fluid (anechoic) filled the whole abdominal cavity even in the retroperitoneal space in all FIP identified cases (Fig. 2d). Thickened intestinal wall with hyper echogenicity was clearly observed, it may be resulted from the severe pyogranulomatous reaction. Mixed hyperechoic patches with diffuse hypo-echogenicity were commonly seen in the liver of most FIP cases. Thick capsule was obviously noticed in spleen and kidney. Hepatic and renal tissues showed minor changes in their echogenicity. Imaging with $\mathrm{X}$ ray revealed that, all FIP cases were presented with loss of serosal details, it was mainly resulted from thick peritoneum due to the extensive inflammatory changes and increased opacity at lower parts of chest cavity due to pleural effusion (Fig. 2e). Discospondylitis was recorded in two cases.

\section{Post-Mortem and Histopathology Finding}

Post-Mortem Findings: All cats presented with FIP clinical signs were monitored until death and autopsy was performed for each case. Thick peritoneal membrane and abdominal effusion with highly viscous fluid were recorded in all confirmed cases. Organomegaly was recorded in liver and spleen. Extensive pyogranulomatous reaction was seen in all serosal surfaces of all internal organs, intestinal tract (Fig. 3a), peritoneal membrane, liver, spleen (Fig. 3b) and even on the exposed abdominal muscles internally with exception of lungs, heart and kidneys, their gross appearance showed less pyogranuloma and only several hemorrhagic patches mixed and whitish necrotic foci were prominent (Fig. 3c).

\section{Histopathological Findings}

Typical histopathological findings (Fig. 4) due to FIP were clearly detected in nine cases and briefly illustrated as follow. The cortical lymphoid follicles and splenic white pulp showed severe lymphoid depletion and accumulation of eosinophilic tissue debris. Renal parenchyma showed diffuse interstitial pyogranulomatous nephritis with replacement of renal tubules by many viable and degenerated neutrophils. The renal pyogranulomatous reaction was accompanied by chronic inflammatory cells including lymphocytes, histiocytes, plump fibroblast and plasma cells. Renal tubules exhibited necrobiotic changes and occasionally existed casts. Liver showed widespread centrilobular necrosis in several hepatic lobules with expansion of the hepatic capsule and abundant eosinophilic necrotic materials. Interestingly, mineralization was noticed around the central veins. Some major blood vessels showed marked vasculitis admixed with abundant mineralization of tunica media alongside intimal hyperplasia led to narrowing of vascular lumen. Severe enteritis was noticed in many circumstances which characterized by mononuclear inflammatory cells infiltration in the lamina propria with marked expansion of the serosal surface. Intestinal sections revealed severe intense pyogranulomatous reaction with heavy fibrinous exudates and numerous neutrophils. Lung showed multifocal granulomatous zones that displayed inflammatory infiltrate which was consisted mainly of lymphocytes beside macrophages with cellular and karyorrhectic debris.
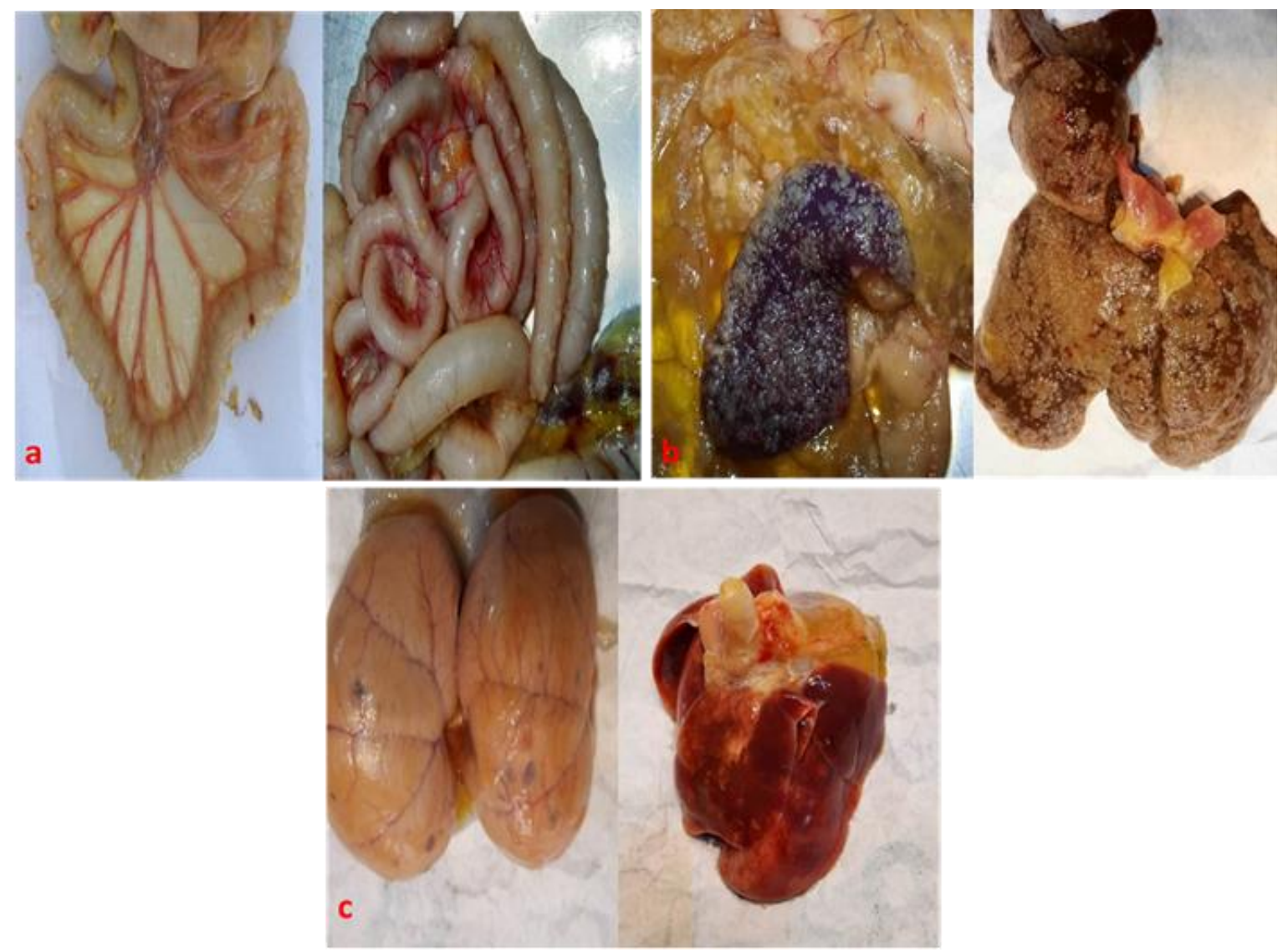

Fig. 3a: Severe pyogranulomatous reaction along the serosal surface of the intestinal tract and associated mesenteric tissues. b: Severe pyogranulomatous reaction with organomegaly in spleen and liver due to FIP in cats. c: Multifocal necrosis with thick capsule in both kidneys and severe congestive pattern in pulmonary tissue due to FIP disease. 

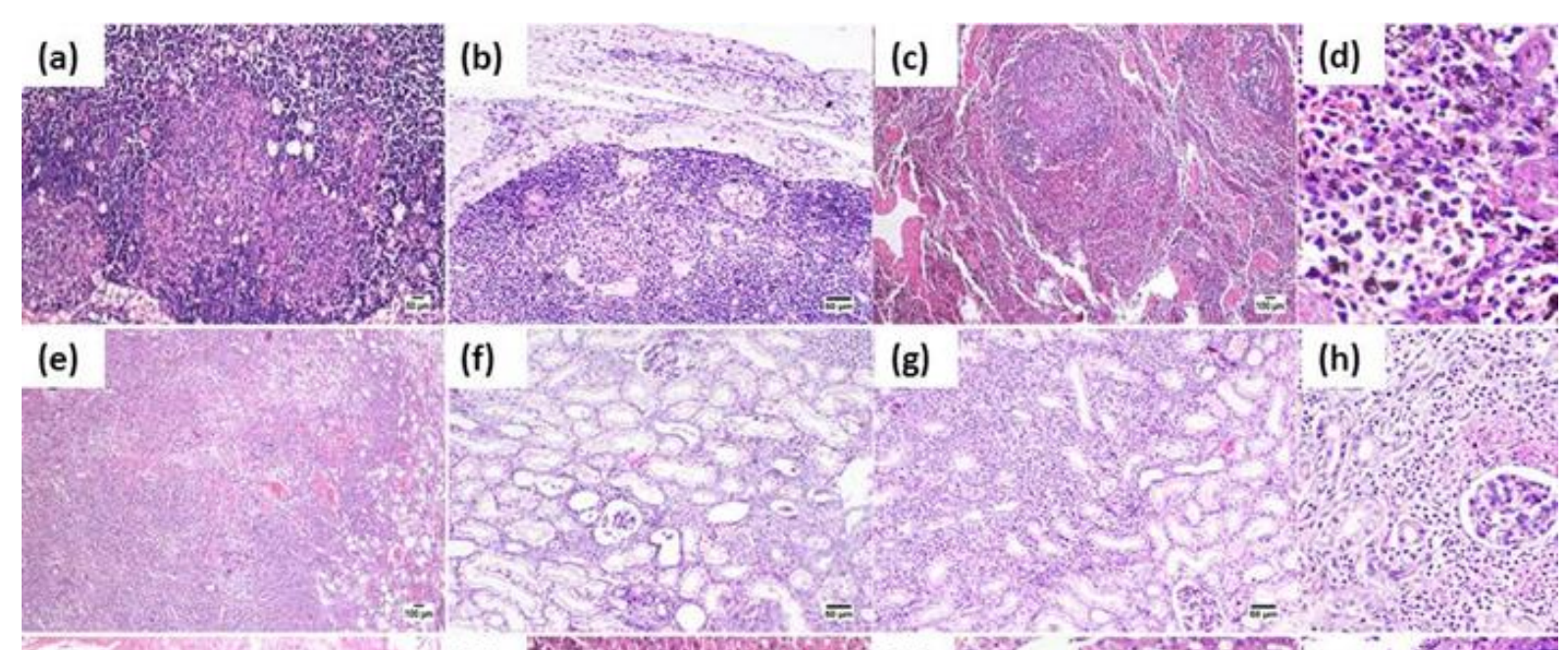

(g)
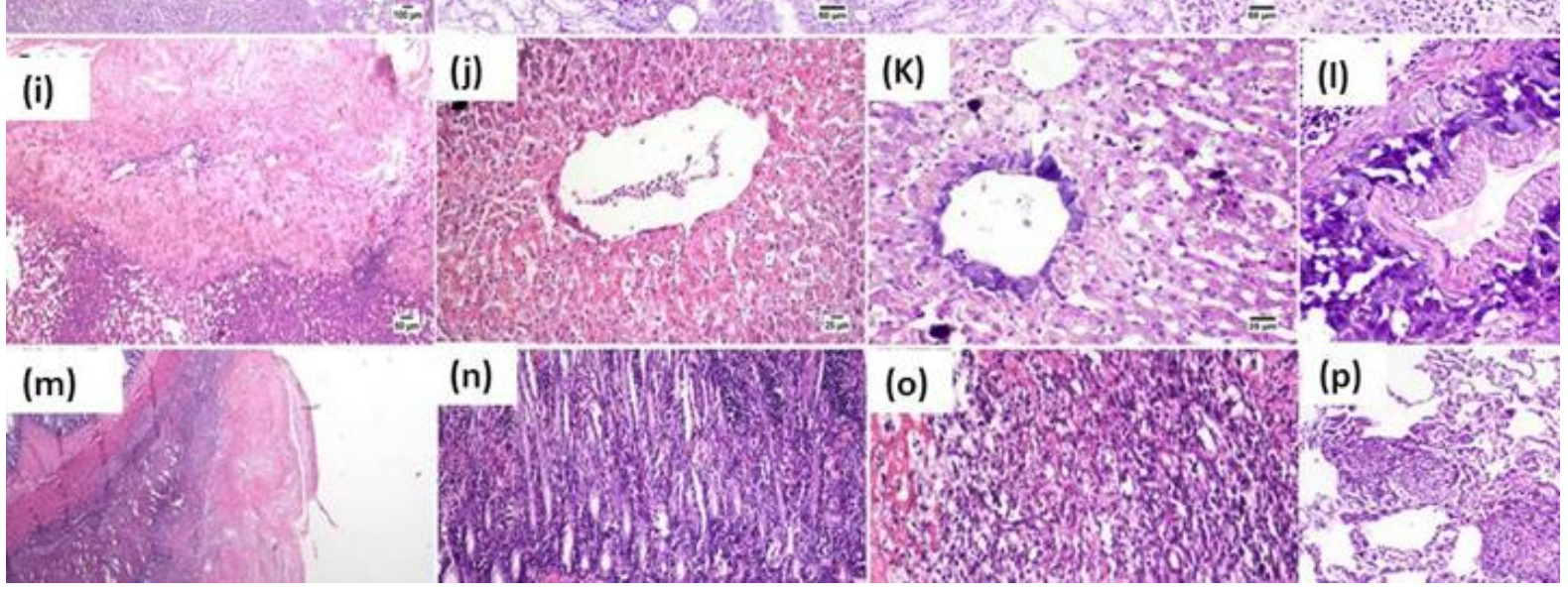

Fig. 4: photomicrograph of feline organs infected with FIP (H\&E). (a) Lymph node showing severe depletion and necrosis of cortical follicles. (b) Spleen showing expansion of the capsule with edema and inflammatory cells infiltration. (c) Spleen showing severe depletion of white pulps. (d) Higher power of splenic red pulp showing precipitation of hemosiderin pigments. (e) Kidney showing end stage kidney illustrated by replacement of renal parenchyma with heavy inflammatory infiltrate. (f) Kidney showing necrosis of renal tubules and presence of casts in the tubular lumen. (g) Kidney showing diffuse interstitial pyogranulomatous nephritis. (h) Higher magnification of renal cortex showing periglomerular infiltration of neutrophils, lymphocytes, and macrophages with accumulation of eosinophilic fibrillar material (fibrinoid necrosis). (i) Hepatic capsule showing severe expansion with eosinophilic proteinaceous exudates. (j) Liver showing centrilobular necrosis. (k) Liver showing necrosis and mineralization around the central vein. (l) Blood vessel showing mineralization of tunica media with intimal hyperplasia. (m) Intestine showing severe expansion of serosal surface with heavy pyogranulomatous and fibrinous exudates. (n) Intestine showing enteritis illustrated by mononuclear cells infiltrating the lamina propria in between the intestinal glands. (o) Higher power of intestine showing many viable and dead neutrophils in submucosa. (p) Lung showing multifocal granulomatous areas that composed of inflammatory cells infiltrate and necrotic debris.

\section{Conclusive Epidemiological Findings}

In line with the results of all diagnostic tests, it was revealed that nearly about $90 \%$ of all examined cats were carrying $\mathrm{FeCoV}$ in their feces. Nearly $5 \%$ of all examined cats (9 out 169) were identified as FIP cases according to the assimilation of results revealed from RT-PCR testing, histopathology, and other used tests. All the confirmed nine cases were presented with wet form and no dry form was detected in the study. The age of the identified FIP cases was above two years old for 6 cases and the other three were below this age. Males and females were nearly equal for susceptibility to the disease, as FIP was identified and confirmed by histopathology in 5 males and 4 females. All males and females identified with FIP were sexually intact. The nine confirmed cases were presented as 6 Persian cats (6/80), 2 mixed breed $(2 / 30)$ and 1 Himalayan (1/19). No FIP cases were detected in the Egyptian Mau cats. Consequently, FIP disease was more prevalent in pure breeds than mixed ones, and the Persian cats were the mostly affected pure breed.

\section{DISCUSSION}

Feline coronavirus $(\mathrm{FeCoV})$ is highly contagious and ubiquitous in multi-cat environment causing mild symptoms or totally an asymptomatic infection (Drechsler et al. 2011). This infection can persist in certain individuals and unpredictably lead to feline infectious peritonitis FIP which is highly fatal systemic immunemediated disease. Regardless the low incidence of FIP disease among the FCoV-infected cats, it is considered as major cause of mortality (Drechsler et al. 2011).

According to the results of screening tests, it is revealed that the infection with $\mathrm{FeCoV}$ was common in the Egyptian multi-cat households as nearly $90 \%$ of studied cats were carrying $\mathrm{FeCoV}$ antigen in their feces, these findings were consistent with worldwide studies related to the $\mathrm{FeCoV}$ infection in cats (Pedersen et al. 2008; Vogel et al. 2010). Most of detected individuals with $\mathrm{FeCoV}$ were asymptomatic and mainly were playing an important role for infection transmission among the healthy cats (Drechsler et al. 2011). 
All clinically suspected cases with FIP were presented with the effusive pattern. The more clinical presentation of FIP disease in the wet form was informed by several reports (Pedersen 2009; Sherding 2009). It may be related to several reasons, firstly, the longer time taken in the dry form to be clinically notable (Kipar and Meli 2014), secondly, may be due to the late transition from dry to wet form before admitting for diagnosis (Kipar and Meli 2014) and finally, existence of less specific clinical symptoms for the dry form which led to high rate of misdiagnosis (Norris et al. 2005).

Diverse of non-specific broad clinical signs were highly reported for FIP disease (Norris et al. 2005) and the same was noted in the current study. Clinical presentation of FIP cases was involving the commonly reported symptoms like persistent fever, lethargy, anorexia with abdominal distension (Hartmann 2005) and further rarely observed ones like the dermatological lesions (Declercq et al. 2008). This clearly reflects high variety for clinical symptoms associated with FIP diseased cats in Egypt.

There are multiple influences which make the definitive diagnosis of FIP extremely difficult like presence of many clinically similar diseases, nonavailable specific test, and more complexity in differentiation between FIP and $\mathrm{FeCoV}$ at the level of diagnosis (Simons et al. 2005; Addie et al. 2009; Sharif et al. 2010). Therefore, the study focused on utilizing of multiple tests to identify FIP disease in suspected cases. Results of immunochromatographic rapid kit for detection of antibodies in serum samples were non-specific as many of clinically healthy cats were seroconverted and showed positive results. These non-specific results clarify the less association between FeCoV antibodies in serum and FIP incidence and this was proven previously (Addie et al. 2009; Sharif et al. 2010).

High sensitivity has been detected with using of Rivalta's testing which is usually performed for the effusive fluid during the clinical diagnosis of wet FIP cases (Hartmann et al. 2003; Felten and Hartman 2019). The similar results were observed during the study, as out of nine histopathological confirmed cases, eight cases showed positive results with Rivalta's test (88.8\%).

Low rate of false positive results plus high specificity was reported with using of RT-PCR for detection of subgenomic mRNA for Feline Coronavirus in blood samples (Simons et al. 2005). The RT-PCR results in the current study showed similar high sensitivity $(8 / 9=88.8 \%)$ and high specificity (93\%).

Laboratory findings of the current study were similar to the hematological results for many of FIP diseased cats in other studies (Addie et al. 2009). They were common in multiple parameters including lymphopenia, variable degrees of non-regenerative anemia and decreased ratio of albumin to globulin (A/G below 0.8). Variability in level of total protein concentration was shown in the FIP cases. Some of them showed hyperproteinemia which resulted from hyperglobulinemia and other cases showed hypoproteinemia which was consequence from chronic alteration of hepatic function (Addie et al. 2009).

Confirmation with histopathology is commonly used for FIP identification and considered as a gold standard for diagnostic test comparisons (Hartmann et al. 2003; Sharif et al. 2010; Giori et al. 2011; SAIDI et al. 2016). It was used for the same purposes in the current study and showed the typical histopathological findings including plasmacellular perivasculitis admixed with inflammatory cells like neutrophils, lymphocytes, and plasma cells. The lymphoid tissues showed lymphoid depletion caused by apoptosis, all these results were identical to different histopathological findings (Haagmans et al. 1996; Kipar et al. 2001; Hartmann et al. 2003; Hartmann 2005).

Post-mortem findings were highly marked by the severe pyogranulomatous reaction in most of internal organs. It was prominent in liver, spleen, intestinal tract, and peritoneal membranes more than kidneys, heart and both lungs. These results may correlate with other findings which stated the viral genome was more detectable in liver and spleen more than kidneys ( $\mathrm{Li}$ and Scott 1994; Sharif et al. 2010).

Different studies suggested that FIP disease has no gender predisposition (Pedersen 1995) and others have suggested that FIP is more prevalent in males (Robison et al. 1971; Schalm 1973; Rohrbach et al. 2001). In the current study, FIP incidence was slightly higher in males than in females and no marked difference was noted between both sexes. All of them were sexually intact and this agreed with previous finding (Rohrbach et al. 2001). Related to breed predisposition studies, the higher incidence for FIP was revealed to be more in the pure breeds and this was similarly detected in the current study. Most of identified FIP cases were aged above two years old and this was inconsistent with several reports which stated that prevalence of FIP disease was higher in age below two years old (Pedersen 1995; Norris et al. 2005).

It was concluded that FIP disease is prevalent in multi-cat household in Egypt, especially in sexually intact pure Persian breed. Further investigations are needed to clarify more epidemiological data about FIP incidence and associated risk factors.

\section{Acknowledgements}

The authors thank the staff members and colleagues of the Department of Medicine and Infectious Diseases, Faculty of Veterinary Medicine, Cairo University, Egypt for their help.

\section{Author's Contribution}

All authors have made significant contributions to the research design, asset and drafted the manuscript. all authors have reviewed and approved the last submitted version.

\section{REFERENCES}

Addie D, Belák S, Boucraut-Baralon C, Egberink H, Frymus T, Gruffydd-Jones T, Hartmann K, Hosie MJ, Lloret A, Lutz H, Marsilio F, Pennisi MG, Radford AD, Thiry E, Truyen $\mathrm{U}$ and Horzinek MC, 2009. Feline infectious peritonitis. $\mathrm{ABCD}$ guidelines on prevention and management. Journal of Feline Medicine and Surgery 11: 594-604. https://doi.org/10.1016/j.jfms.05.008

Balint A, Farsang A, Zadori Z, Hornyak A, Dencso L, Almazan F, Enjuanes L and Belak S, 2012. Molecular characterization of feline infectious peritonitis virus strain df-2 and studies of the role of orf $3 \mathrm{abc}$ in viral cell tropism. Journal of Virology 86: 6258-6267. 
Bank-Wolf BR, Stallkamp I, Wiese S, Moritz A, Tekes G and Thiel HJ, 2014. Mutations of $3 \mathrm{c}$ and spike protein genes correlate with the occurrence of feline infectious peritonitis. Veterinary Microbiology 173: 177-188. https://doi.org/ 10.1016/j.vetmic.2014.07.020

Barker EN, Stranieri A, Helps CR, Porter EL, Davidson AD, Day MJ, Knowles T, Kipar A and Tasker S, 2017. Limitations of using feline coronavirus spike protein gene mutations to diagnose feline infectious peritonitis. Veterinary Research 48: 60. https://doi.org/10.1186/ s13567-017-0467-9

Borschensky CM and Reinacher M, 2014. Mutations in the 3c and $7 \mathrm{~b}$ genes of feline coronavirus in spontaneously affected FIP cats. Research in Veterinary Science 97: 333340. https://doi.org/10.1016/j.rvsc.2014.07.016

Brown MA, Troyer JL, Pecon-Slattery J, Roelke ME and O'Brien SJ, 2009. Genetics and pathogenesis of feline infectious peritonitis virus. Emerging Infectious Disease 15: 1445-1452. https://doi.org/10.3201/eid1509.081573

Chang HW, de Groot RJ, Egberink HF and Rottier PJ, 2010. Feline infectious peritonitis: insights into feline coronavirus pathobiogenesis and epidemiology based on genetic analysis of the viral $3 \mathrm{c}$ gene. Journal of General Virology 91: 415-420.

Chang HW, Egberink HF, Halpin R, Spiro DJ and Rottier PJ, 2012. Spike protein fusion peptide and feline coronavirus virulence. Emerging Infectious Disease 18: 1089-1095.

Chauhan RS and Agarwal DK, 2008. Histopathology as an aid in diagnosis, In: Textbook of Veterinary, Clinical, and Laboratory Diagnosis ( $2^{\text {nd }}$ Ed). Jaypee Brothers Publishers.

Declercq J, De Bosschere H, Schwarzkopf I and Declercq L, 2008. Papular cutaneous lesions in a cat associated with feline infectious peritonitis. Veterinary Dermatology 9: 255-8. https://doi.org/10.1111/j.1365-3164.2008.00684.x

Dedeurwaerder A, Desmarets LM, Olyslaegers DA, Vermeulen BL, Dewerchin HL and Nauwynck HJ, 2013. The role of accessory proteins in the replication of feline infectious peritonitis virus in peripheral blood monocytes. Veterinary Microbiology 162: 447-455.

Desmarets LM, Vermeulen BL, Theuns S, Conceicao-Neto N, Zeller M, Roukaerts ID, Acar DD, Olyslaegers DA, Van Ranst M and Matthijnssens J, 2016. Experimental feline enteric coronavirus infection reveals an aberrant infection pattern and shedding of mutants with impaired infectivity in enterocyte cultures. Scientific Reports 6: 20- 22.

Dewerchin HL, Cornelissen E and Nauwynck HJ, 2005. Replication of feline coronaviruses in peripheral blood monocytes. Archive Virology 150: 2483-2500.

Drechsler Y, Alcaraz A and Bossong FJ, 2011. Feline coronavirus in multi cat environments. Veterinary Clinics of North America: Small Animal Practice 41: 1133-1169.

Felten S and Hartmann K, 2019. diagnosis of feline infectious peritonitis: a review of the current literature. Viruses 11: 1068-1105.

Giori L, Giordano A, Giudice C, Grieco V and Paltrinieri S, 2011. Performances of different diagnostic tests for feline infectious peritonitis in challenging clinical cases. Journal if Small Animal Practice 52: 152-7. https://doi.org/10.1111/ j.1748-5827.2011.01042.x

Haagmans BL, Egberink HF and Horzinek MC, 1996. Apoptosis and T-cell depletion during feline infectious peritonitis. Journal of Virology 70: 8977-8983.

Hartmann K, 2005. Feline infectious peritonitis. Veterinary Clinics of North America: Small Animal Practice 35: 3979. https://doi.org/10.1016/j.cvsm.2004.10.011

Hartmann K, Binder C and Hirschberger J, 2003. Comparison of different tests to diagnose feline infectious peritonitis. Journal of Veterinary Internal Medicine 17: 781-790.
Heald-Sargent T and Gallagher T, 2012. Ready, set, fuse! The coronavirus spike protein and acquisition of fusion competence. Viruses 4: 557-580.

Hora AS, Tonietti PO, Taniwaki SA, Asano KM, Maiorka P, Richtzenhain LJ and Brandao PE, 2016. Feline coronavirus $3 \mathrm{c}$ protein: A candidate for a virulence marker? BioMed Research International, 8560691.

Howell M, Evans SJM, Cornwall M and Santangelo KS, 2020. Multiplex fluorescent immunocytochemistry for the diagnosis of feline infectious peritonitis: Determining optimal storage conditions. Veterinary Clinical Pathology 49: 640-645. https://doi.org/10.1111/vcp.12912

Jaimes JA and Whittaker GR, 2018. Feline coronavirus: Insights into viral pathogenesis based on the spike protein structure and function. Virology 517: 108-121.

Jolanda DF, Groot M, Jessica M, Robbert G and Raoul J, 2005. Natural history of a recurrent feline coronavirus infection and the role of cellular immunity in survival and disease. Journal of Virology 79: 1036-1044.

Kass PH and Dent TH, 1995. The epidemiology of feline infectious peritonitis in catteries. Feline Practice 23: 27-32.

Kipar A, Kohler K and Leukert W, 2001. A comparison of lymphatic tissues from cats with spontaneous feline infectious peritonitis (FIP) cats with FIP virus infection but no FIP, and cats with no infection. Journal of Comparative Pathology 125: 182-191.

Kipar A, May H, Menger S, Weber M, Leukert W and Reinacher M, 2005. Morphologic features and development of granulomatous vasculitis in feline infectious peritonitis. Veterinary Pathology 42: 321-330.

Kipar A and Meli ML, 2014. Feline infectious peritonitis: still an enigma. Veterinary Pathology 51: 505-26. https://doi.org/ $10.1177 / 0300985814522077$

Kiss I, Kecskemeti S, Tanyi J, Klingeborn B and Belak S, 2000. Preliminary studies on feline coronavirus distribution in naturally and experimentally infected cats. Research in Veterinary Science 68: 237-242.

Levy JK, Crawford PC, Tucker SJ, 2017. Performance of 4 point-of-care screening tests for feline leukemia virus and feline immunodeficiency virus. Journal of Veterinary Internal Medicine 31: 521-526. https://doi.org/10.1111/ jvim. 14648

Lewis CS, Porter E, Matthews D, Kipar A, Tasker S, Helps CR and Siddell SG, 2015. Genotyping coronaviruses associated with feline infectious peritonitis. Journal of General Virology 96: 1358-1368.

Li X and Scott FW, 1994. Detection of feline coronaviruses in cell cultures and in fresh and fixed feline tissues using polymerase chain reaction. Veterinary Microbiology 42: 65-77. https://doi.org/10.1016/0378-1135(94)90078-7

Meli M, Kipar A, Muller C, Jenal K, Gonczi E, Borel N, GunnMoore D, Chalmers S, Lin F and Reinacher M, 2004. High viral loads despite absence of clinical and pathological findings in cats experimentally infected with feline coronavirus (fcov) type $\mathrm{i}$ and in naturally fcov-infected cats Journal of Feline Medical Surgery 6: 69-81.

Michimae Y, Mikami S, Kazuo O, Toyosawa K, Matsumoto I, Kouchi M, Koujitani T, Inoue T and Seki T, 2010. The first case of feline infectious peritonitis-like pyogranuloma in a ferret infected by coronavirus in Japan. Journal of Toxicology and Pathology 23: 99-101.

Norris JM, Bosward KL, White JD, Baral RM, Catt MJ and Malik R, 2005. Clinicopathological findings associated with feline infectious peritonitis in Sydney, Australia: 42 cases (1990-2002). Australian Veterinary Journal 83: 666673.

Pedersen NC, 1995. An overview of feline enteric coronavirus and infectious peritonitis virus infections. Feline Practice 23: 7-20. 
Int J Vet Sci, 2022, 11(2): 159-167.

Pedersen NC, Allen CE and Lyons LA, 2008. Pathogenesis of feline enteric coronavirus infection. Journal of Feline Medicine and Surgery 10: 529-541.

Pedersen NC, Liu H, Scarlett J, Leutenegger CM, Golovko L, Kennedy $\mathrm{H}$ and Kamal FM, 2012. Feline infectious peritonitis: Role of the feline coronavirus $3 \mathrm{c}$ gene in intestinal tropism and pathogenicity based upon isolates from resident and adopted shelter cats. Virus Research 165: $17-28$.

Pedersen NC, 2009. A review of feline infectious peritonitis virus infection: 1963-2008. Journal of Feline Medicine and Surgery 11: 225-258.

Porter E, Tasker S, Day MJ, Harley R, Kipar A, Siddell SG and Helps CR, 2014. Amino acid changes in the spike protein of feline coronavirus correlate with systemic spread of virus from the intestine and not with feline infectious peritonitis Veterinary Research 45: 49-55.

Robison RL, Holzworth J and Gilmore CE, 1971. Naturally occurring infectious peritonitis: signs and clinical diagnosis. Journal of the American Veterinary Medical Association 158: 981-986.

Rohrbach BW, Baldwin CA and Reed WM, 2001. Epidemiology of feline infectious peritonitis among cats examined at veterinary medical teaching hospitals. Journal of the American Veterinary Medical Association 218:1111-1115.

Rottier PJ, Nakamura K, Schellen P, Volders H and Haijema BJ, 2005. Acquisition of macrophage tropism during the pathogenesis of feline infectious peritonitis is determined by mutations in the feline coronavirus spike protein Journal of Virology 79: 14122-14130.

Saidi S, Safi S, Mashhadi Rafii S, Kabir FR and Mortazavi P, 2016. Comparison of ELISA test with histopathology in the diagnosis of feline infectious peritonitis. Journal of Comparative Pathobiology 13: 1955-1964.

Schalm OW, 1973. Feline infectious peritonitis: vital statistics and laboratory findings. Feline Practice 3: 18-20.

Sharif S, Arshad SS, Hair-Bejo M, Omar AR, Zeenathul NA and Alazawy A, 2010. Diagnostic methods for feline coronavirus: a review. Veterinary Medicine International https://doi.org/10.4061/2010/809480

Sherding RG, 2006. Feline Infectious Peritonitis (Feline Coronavirus). Saunders Manual of Small Animal Practice 132-43. https://doi.org/10.1016/B0-72-160422-6/50012-7

Simons FA, Vennema H, Rofina JE, Pol JM, Horzinek MC, Rottier PJ and Egberink HF, 2005. A mRNA PCR for the diagnosis of feline infectious peritonitis. Journal of Virology Methods 124: 111-116.

Sparkes AH, 2004. "Feline coronavirus infection," in Feline Medicine and Therapeutics, Blackwell publishing, Oxford, UK, 3rd edition. pp: 623-634

Tekes G and Thiel HJ, 2016. Feline Coronaviruses: Pathogenesis of Feline Infectious Peritonitis. Advances in virus research 96: 193-218. https://doi.org/10.1016/bs.aivir.2016.08.002

Vogel L, Van der Lubben M, te Lintelo EG, Bekker CP, Geerts T, Schui LS, Grinwis GC, Egberink HF and Rottier PJ, 2010. Pathogenic characteristics of persistent feline enteric coronavirus infection in cats. Veterinary Research 41: 71. 